\title{
A Navigation Algorithm Inspired by Human Navigation
}

\author{
Vijesh M \\ intern \\ Indian Statistical Institute \\ Chennai, India \\ mv.vijesh@gmail.com
}

Amitash Ramesh
Intern
Indian Statistical Institute
Chennai, India
amitashr@gmail.com

\author{
Vijay Mahantesh SM \\ Intern \\ Indian Statistical Institute \\ Chennai, India \\ vijaym123@gmail.com
}

\begin{abstract}
Human navigation has been a topic of interest in spatial cognition from the past few decades. It has been experimentally observed that humans accomplish the task of way-finding a destination in an unknown environment by recognizing landmarks. Investigations using network analytic techniques reveal that humans, when asked to way-find their destination, learn the top ranked nodes of a network. In this paper we report a study simulating the strategy used by humans to recognize the centers of a network. We show that the paths obtained from our simulation has the same properties as the paths obtained in human based experiment. The simulation thus performed leads to a novel way of path-finding in a network. We discuss the performance of our method and compare it with the existing techniques to find a path between a pair of nodes in a network.
\end{abstract}

\section{INTRODUCTION}

The problem of finding a target vertex in a network where one is provided with just the local information is a well studied problem. Starting from the work of Milgram in 1967 22], the current methods include routing using full-tables 12 , interval routing 11, 12, routing labeling schemes 23, 26, greedy routing 14, 20, geographic routing 14, compass routing 14], etc., These routing techniques are mainly used in transportation networks and in wired as well as wireless communication networks. For detailed survey one can refer to $11,12,14$ and 23 .

For surveys of the routing methods in social networks and email networks in particular, one is referred to $2,3,10,20$, 21

In the classical small world experiment by Milgram 22], the letter from Nebraska was to reach Boston. This is a scenario

\author{
Veni Madhavan \\ Computer Science and \\ Automation \\ Indian Institute of Science \\ Bangalore, India
}

where the sender actively needs to participate in the process of helping the letter reach the destination, while the receiver remains passive. Let us consider a variation of this problem where both sender and receiver participate in the process of finding a path between them.

\section{Motivation}

Sudarshan et al., in 16 presents a network analytic approach to understand human navigation. Their experiment comprises of making human participants navigate on a network that is unknown to them. Participants are given a source node and are asked to navigate to a destination node. The underlying network is not visible to the participant in its entirety. At any instance, participant can view the adjacent nodes of the node that $\mathrm{s} /$ he is currently present. Several such source destination node pairs are given to the participant. The time taken and the path taken are recorded.

They observe that participants eventually learn a strategy which helps them navigate easily and quickly. They show that the participants learn a few landmark nodes which they use as via media to navigate. They further show that these landmark nodes have superior closeness-centrality ranking.

In the current paper, we present a navigation algorithm based on the strategy used by human participants in the experiment reported in 16].

The strategy of human participants in the above mentioned experiment to learn centers of a network and the pursuit of paths through the centers has motivated the proposal of this novel navigation algorithm.

\section{The Technique}

In an unweighted graph $G(V, E)$, given a source vertex $s$ and a destination vertex $t$, a straight forward approach to establish a path between $s$ and $t$ is to take a random walk from $s$ till we reach $t$. One way to better this random walk method is to take a two way random walk, one from the source $s$ and the other from the destination $t$ and stop once the two random walks intersect. This method is expected to be quicker than the first in terms of number of hops that is required to establish a path. We provide empirical results 
to support this.

We describe our algorithm (path concatenation algorithm or PCA) and compare it with two other path finding strategies based on random walks. We apply PCA on various synthetic networks and discuss its performance. We show that PCA yields center-strategic paths on scale free networks - as was the case with the paths that the participants took in the two experiment.

We start with the description of related work in Section. 2 and then in Section. 3 present the notations and notions used. Section. comprises of the application of the described algorithm on synthetic networks. We benchmark our results by comparing them with two other navigational techniques. In Section. 7 we conclude with a possible further improvements.

\section{RELATED WORK}

Kleinberg's work 18 on navigation in a small world, was the first paper to shed light on the navigation problem on complex networks. The paper highlighted the fact that it is easier to find short chains between points in some networks than others.

Adamic et al., in 3 introduced several local search strategies to find a path to the given destination vertex. Their strategies are limited to applications on networks that obey the power law. The results are shown on GNUTELLA peer-topeer network which is known to obey power law. In 17], Kim et al., numerically compares the local path finding strategies with that of global path finding strategies on scale free networks.

A rigorous graph theory based approach to path finding strategy was proposed by Gavoille et al., [13. They provide a method of navigation based on vertex labeling. They establish a labeling strategy for the vertices of a graph in a way that allows one to compute the distance between any two vertices directly from the labels, without using any additional information about the network.

Adamic et al., in 1] addresses the question of how participants in a small world experiment are able to find short paths in a social network using only local information about their immediate contacts. They conduct their experiment on an email network and demonstrate by empirical data that the small world search strategies using a contact's position in physical space or in an organizational hierarchy relative to the target can effectively be used to locate most individuals.

A detailed survey on decentralized search algorithms on networks that exhibit small-world phenomena is given by Kleinberg in 19. This survey also contains an exhaustive list of open problems in this area.

Ozgur et al., 25 propose a navigational technique based on a hybridized method of using degree and homophilly of vertices that yield better results than the currently known techniques.

Cajueiro et al., in 6] proposed a learning framework based on a First-Visit Monte Carlo Algorithm. They show that the navigation difficulty and learning velocity are strongly related to the network topology. In [5], Cajueiro has proposed a strategy where the walker is assumed to take optimal paths in order to minimize the cost of walking. They provide an approach to generalize several concepts presented in the literature concerning random navigation and direct navigation.

Recently a novel method of navigating a network using the underlying spanning tree was proposed by Dragan et al. in 8 . The paper provides a thorough graph theoretic treatment of the navigation problem. They reduce the navigation problem on a graph $G$ to that of the underlying spanning tree and navigate on the latter.

On the application front, such navigational techniques are useful in a problem of finding paths between vertices in peerto-peer systems. Crespo et al., 7] in their work on routing indices on Peer-to-Peer systems, propose a novel method of query forwarding from vertices to their neighbors that are more likely to have answers. They provide different novel routing schemes and evaluate their performance.

\section{NOTATIONS AND NOTIONS}

A graph $G=(V, E)$ is composed of a set of nodes $V$ and a set of edges $E \subseteq V \times V$, with $|V|=n$ and $|E|=m$. A way between two nodes $u$ and $v$ is any sequence of edges $\left(e_{1}, e_{2}, \ldots, e_{k}\right)$ with $e_{1}=\left(u, x_{1}\right), e_{2}=\left(x_{1}, x_{2}\right), \ldots, e_{k}=$ $\left(x_{k-1}, v\right)$. A path is a way with no repeating nodes. The length of a way is defined as the number of edges in it. A shortest path between two nodes $u$ and $v$ is a path with length $l$, such that, every other path between $u$ and $v$ is of length greater than or equal to $l$. Two nodes are said to be connected if there exists a path between them. The distance $d\left(v_{1}, v_{2}\right)$ between any two nodes is defined as the length of a shortest path between them, or set to $\infty$ if there exists no path between them. Any maximal set of pairwise connected nodes is called a component of the graph. Given $v \in V$, $N_{G}(v)$ denotes the set of all adjacent vertices of $v$.

Let $W_{u}=\left(u=v_{0}, v_{1}, v_{2}, \ldots\right)$ denote a random walk starting from the vertex $u$. By $W_{u}[i]$ we mean the vertex at the $i$ th step of the random walk $W_{u}$. e.g. $W_{u}[2]=v_{2}$. Let $T\left(W_{u}, i\right)$ denote the set $T\left(W_{u}, i\right)=\left\{v_{0}, v_{1}, \ldots, v_{i}\right\}$ of all vertices that are being visited by the random walk $W_{u}$ during the first $i$ steps.

A centrality index is a real-valued function $C: V \rightarrow \mathbb{R}$ on the nodes. The intuition is that the higher the value of this function, the more central this node is for the network. There are various indices; in this article we use the so-called closeness centrality 24] $C_{C}(v)$, which is defined as the reciprocal of sum of the distances of $v$ to all other nodes $w$ :

$$
C_{C}(v)=1 / \sum_{w \in V} d(v, w)
$$

For any given graph, a centrality index can be used to define a ranking on the nodes, by sorting the nodes non-decreasingly by their centrality value. Ties if any, are broken arbitrarily. we denote the rank of a vertex by $R(v)$.

Given a walk from $s$ to $t$, it is easy to see that one can get a path from $s$ to $t$ on this walk by removing the cy- 
cles. (Refer 15]).By $P\left(W_{u}, i\right)$ we mean a path obtained by considering the first $i$ steps taken by the random walk $W_{u}$.

Through out the paper, we assume that the graph is an undirected simple graph, unless otherwise stated.

\section{THE ALGORITHM}

The path concatenation algorithm is split into two components.

- The Learning Phase

- The Navigation Phase

The learning phase deals with the preprocessing of the graph G. Once the preprocessing phase is complete, the navigation phase describes how one can find a path between a given pair of vertices.

\subsection{Learning Phase}

In the learning phase, the undirected graph $G(V, E)$ is converted to a directed weighted graph $G_{d}\left(V, E_{d}\right)$

Let $u, v \in_{r} V$ be two randomly picked vertices. Consider the random walks $W_{u}$ and $W_{v}$. As defined above, let $W_{u}=$ $\left(W_{u}[0], W_{u}[1], \ldots\right)$ and $W_{v}=\left(W_{v}[0], W_{v}[1], \ldots\right)$.

Consider least $i$ such that $T\left(W_{u}, i\right) \cap T\left(W_{v}, i\right) \neq \emptyset$.

Such an $i$ guarantees a vertex $h \in T\left(W_{u}, i\right) \cap T\left(W_{v}, i\right)$. Clearly, one can construct a path from $u$ to $h$ and another path from $v$ to $h$. Let us call these directed paths $P_{u, h}$ and $P_{v, h}$ respectively. Let $l\left(P_{u, h}\right)$ and $l\left(P_{v, h}\right)$ denote the respective path lengths.

We define a function on the vertex set called the Vertex Reward function and a function on the edge set called the Edge Reward function as follows.

\subsubsection{Vertex Reward}

Consider a function flag: $V_{d} \rightarrow \mathbb{Z}$, where $\operatorname{flag}(v)=0, \forall v \in$ $V_{d}$ to begin with. In an iteration, we pick a random pair of vertices $u$ and $v$, and take random walks $W_{u}$ and $W_{v}$. Once the walks intersect at a vertex, say $h$, we increment $f l a g(h)$ by 1 , i.e. $f \operatorname{lag}(h)=\operatorname{flag}(h)+1$.

\subsubsection{Edge Reward}

Given the graph $G(V, E)$, at the end of the learning phase, we need to obtain a weighted directed graph $G\left(V, E_{d}\right)$, with $E_{d}=V \times V$. We define a reward function $R: E_{d} \rightarrow \mathbb{R}$. We first initialize $R(u, v)=0, \forall u, v \in V_{d}$. For every edge $(a, b)$ belonging to the path $P_{u, h}$, we increment the reward of that edge by $R(a, b)=R(a, b)+\frac{1}{l\left(P_{u, h}\right)}$. Similarly for all edges $(c, d)$ belonging to the path $P_{v, h}$, we increment the reward of the edge $(c, d)$ by $R(c, d)=R(c, d)+\frac{1}{l\left(P_{v, h}\right)}$.

Below is the pidgin code of the algorithm.
4.1.3 Algorithm for Learning Phase

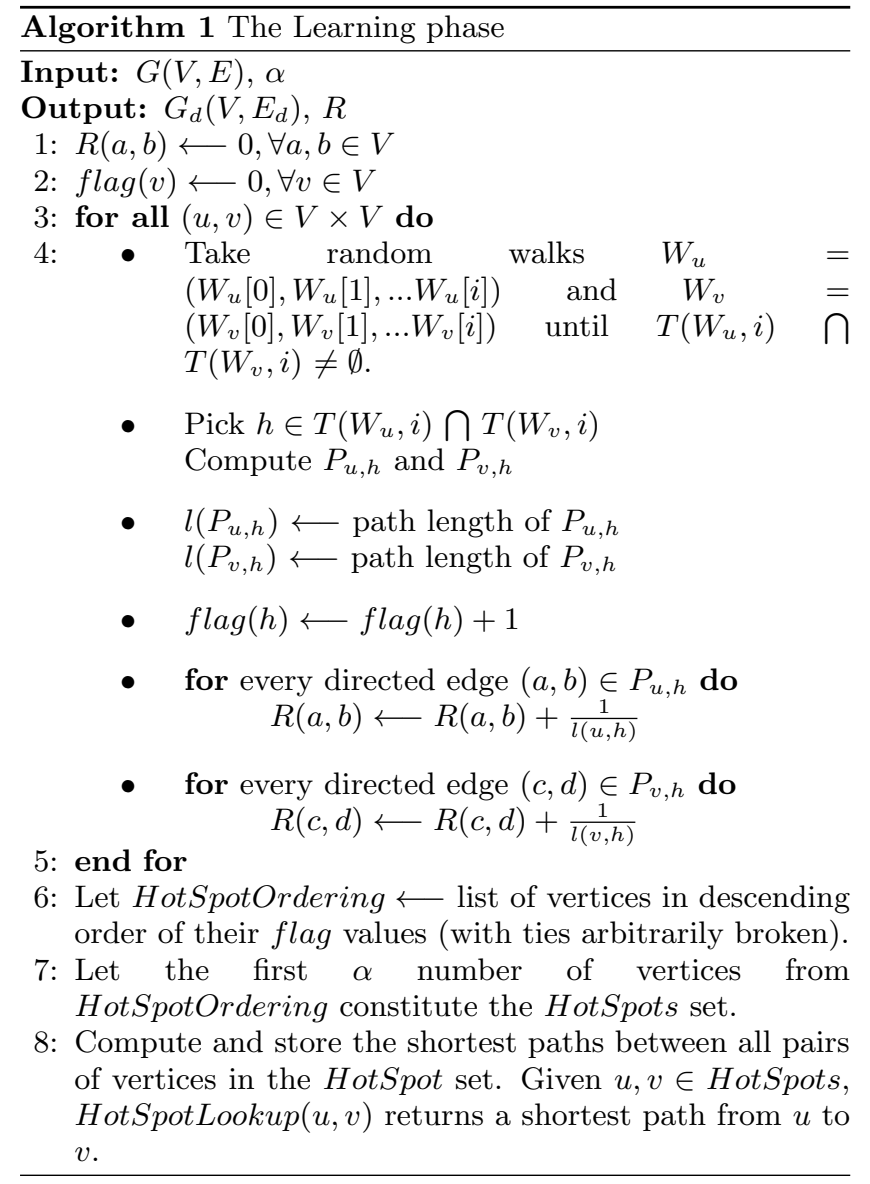

Algorithm 4.1 .3 is a pre-processing phase where, if a graph $G(V, E)$ is given as input, one obtains a directed weighted graph and a table of values comprising of shortest paths between $\alpha$ number of top flag valued vertices of the graph, i.e., all pairs shortest paths amongst the vertices in HotSpots.

We now describe how one can find a path from a given source vertex $s$ to a destination vertex $t$ by making use of the weights in the directed weighted graph thus obtained. Information from HotSpots, HotSpotLookup and the edge reward function $R$ is utilized to find a path from a source to a destination vertex.

Figure. 1 illustrates the learning phase of Algorithm 4.1.3 $u$ and $v$ are the chosen pair of vertices. The red walk from $u$ and the green walk from $v$ indicate the random walks $W_{u}$ and $W_{v}$ respectively. $h$ is the vertex at which the intersection has occurred. The blue path from $u$ and the pink path from $v$ indicate $P_{u, h}$ and $P_{v, h}$ respectively. $l\left(P_{u, h}\right)$ and $l\left(P_{v, h}\right)$ are the lengths of the blue and pink path respectively. The directed edges along the blue path and the pink path are rewarded with $\frac{1}{l\left(P_{u, h}\right)}$ and $\frac{1}{l\left(P_{v, h}\right)}$ respectively. flag $(h)$ is incremented by 1 .

\subsection{Navigation Phase}

The navigation phase makes use of the output $G_{d}\left(V, E_{d}\right)$ and the set $R$, which contains values of the edges obtained in the learning phase to find a path between a given source 


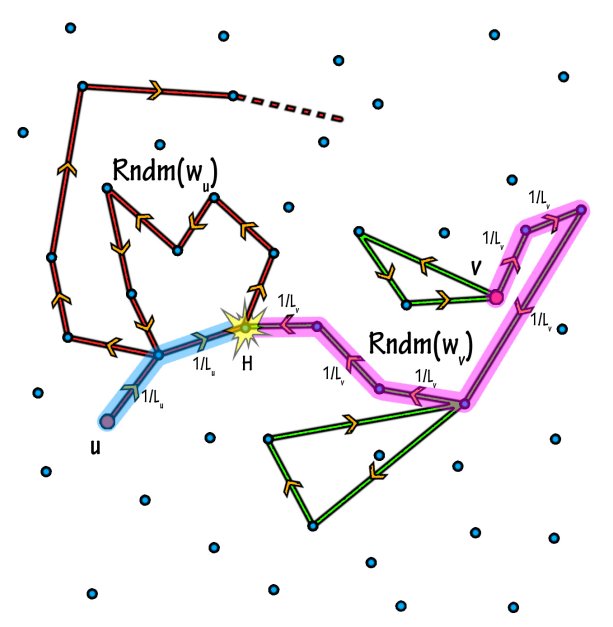

Figure 1: The Learning Phase

vertex $s \in V$ to a destination vertex $t \in V$. The technique involves 3 stages:

1. Finding a path from $s$ to $h_{s}$, where $h_{s} \in$ HotSpots

2. Finding a path from $t$ to $h_{t}$, where $h_{t} \in$ HotSpots

3. Concatenating the paths $s$ to $h_{s}, h_{s}$ to $h_{t}$ and $h_{t}$ to $t$.

The paths $s$ to $h_{s}$ and $t$ to $h_{t}$ are constructed by choosing adjacent edges with the highest $R$ value. We call this the greedy traversal technique. This is illustrated in Figure 2. Assuming that we start from a start vertex $s=u$, let $u_{0}$, $u_{1}, u_{2} u_{3}$ and $u_{4}$ denote the neighbors of $u$. As can be seen in Figure 2, the edge with the highest $R$ value is $\left(u, u_{1}\right)$, we choose this as the edge and continue forming a path by continuing this process $\left(\left(u_{1}, u_{11}\right)\right.$ is chosen next).

The paths thus obtained are finally concatenated to get a path from $s$ to $t$. See Figure 3 for illustration.

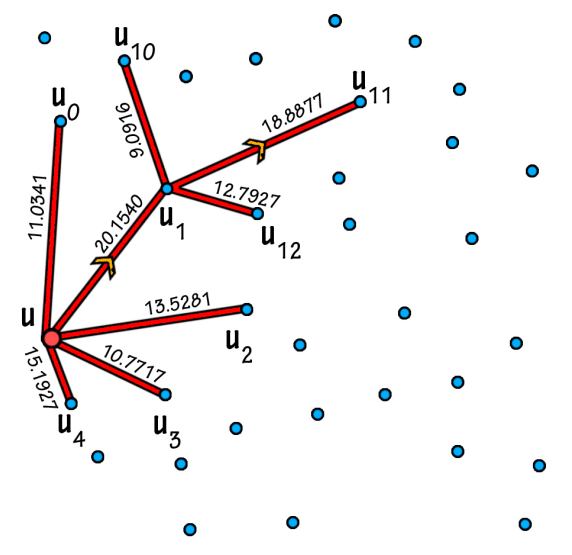

Figure 2: Greedy Traversal Technique

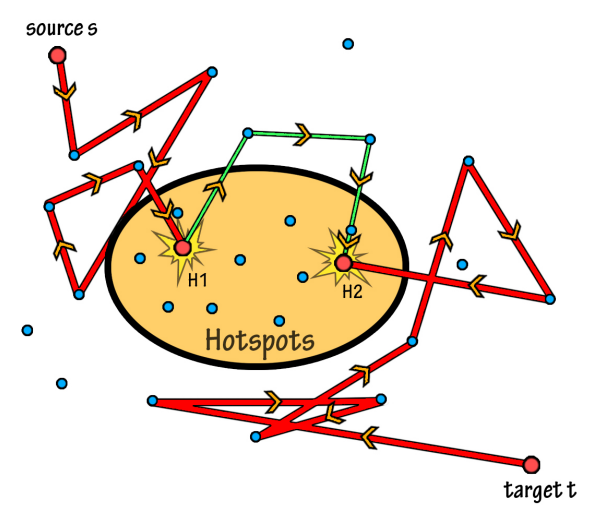

Figure 3: The Navigation Phase

\section{RESULTS AND DISCUSSIONS}

We discuss in this section, the results that were obtained when the path concatenation algorithm was applied to several classes of graphs.

We mainly test our results on two types of synthetic networks: Barabasi-Albert (scale-free) graphs and Erdos-Renyi graphs.

Barabasi-Albert graphs: Barabasi-Albert Graphs [4] are a class of graphs constructed by preferential attachment of vertices.

Erdos-Renyi graphs: Erdos-Renyi Networks 9] are a class of graphs constructed by including the edges with a given probability $p$.

\subsection{Selection of optimum value of $\alpha$}

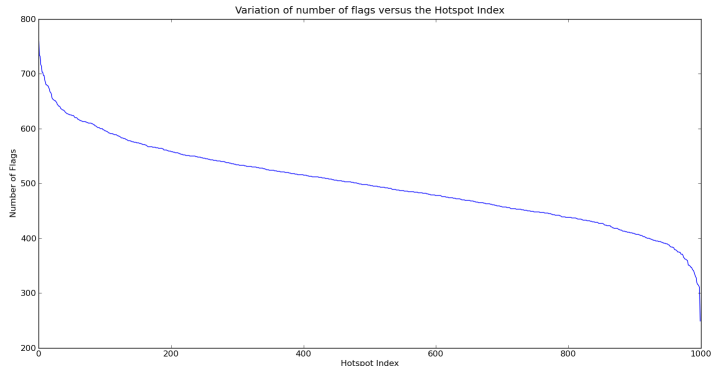

Figure 4: Plot of variation of number of flags versus HotSpotIndex for an Erdos-Renyi network of $\mathbf{1 0 0 0}$ vertices and edge probability of 0.15

The HotSpotOrdering list contains the vertex labels arranged in the decreasing order of their number of flags. Let HotSpotIndex $(u)$ denote the position of $u$ in HotSpotOrdering.

Let HotSpotOrdering $=\left\{h_{1}, h_{2}, h_{3}, \ldots . h_{|V|}\right\}$.

Let HotSpotIndex $\left(h_{i}\right)=i$. 


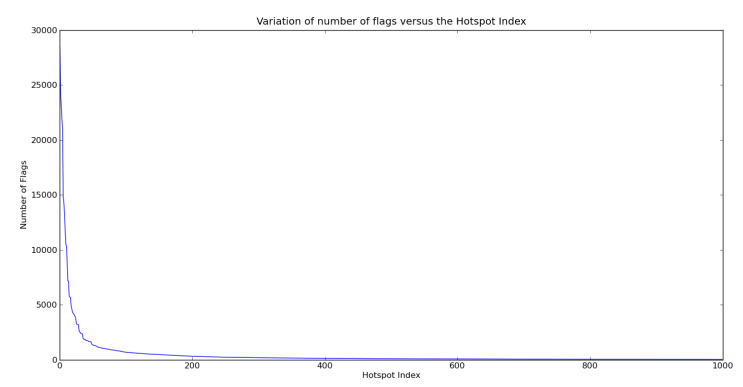

Figure 5: Plot of variation of number of flags versus HotSpotIndex for a Scale Free Graph of 1000 vertices and 4 connections.

The plots in Figure. 4 and 5 represents a variation of flag values Vs HotSpotIndex.

Let $\alpha=\mid$ HotSpots $\mid$. As $\alpha$ decreases, the navigation through the network during the navigation phase (Section. 4.2) becomes increasingly difficult since it takes a longer path to reach vertices in HotSpots. As $\alpha$ increases, the difficulty involved in computing the all pairs shortest path between the elements of HotSpots using Djikstra's Algorithm increases cubically, $O\left(\alpha^{3}\right)$. There is thus a need to determine an optimal value for $\alpha$.

By analyzing the plot of number of flags versus HotSpotIndex, we see that the curve takes a sharp turn at the initial values of HotSpotIndex in the above figures. This transition clearly shows that there are some vertices in the graph with relatively higher importance. That is, the rate of decrease of flagging decreases drastically at the point where the curve takes a sharp turn. Further addition of vertices into HotSpots doesn't contribute significantly towards improving the efficiency of our algorithm. In fact, it causes a computational overhead during the creation of HotSpotLookup. Hence, from this point onwards, further addition of the vertices corresponding to HotSpotIndex to HotSpots becomes redundant. Hence, we set $\alpha$ to a value of the curve at which it takes a sharp turn (i.e. a drastic slope change value).

Figure. 4 illustrates the flag distribution for an Erdos-Renyi network of 1000 vertices and an edge probability of 0.15 . The plot shown here takes a sharp turn at the 33rd hotspot. Hence, $\alpha$ is set to 33. Figure. 5 illustrates the flag distribution for a scale-free graph of 1000 vertices and 4 connections. The plot shown here takes a sharp turn at the 100th hotspot. Hence, $\alpha$ is set to 100 .

\subsection{Comparison Between Various Navigation Techniques}

This section highlights the effectiveness of greedy navigation over other methods of navigation. Consider two vertices $s, t \in V$. Let $s$ be source and $t$ be the target vertex. In the navigation phase of the algorithm, our aim is to establish a path between $s$ and $t$. Let $d(s, t)$ denote the length of the shortest path between $s$ and $t$. Here are a few methods one can adopt to accomplish the task of establishing a path between $s$ and $t$ :

\subsubsection{1-Way Random Walk}

The idea here is to start from the source vertex $s$ and take a random walk $W_{s}$ until the target vertex $t$ is reached. Since this technique involves a single random walk, we refer to it as a 1-way random walk. Let $\beta_{s, t}$ denote the path length of the path corresponding to the random walk $W_{s}$ from $s$ to $t$. Let $\beta$ be the average ratio of length of 1-way random walk and length of the shortest path, taken over all unordered vertex pairs $(s, t)$, such that $s, t \in V$.

$$
\beta=\frac{1}{\left(\begin{array}{c}
|V| \\
2
\end{array}\right)} \sum_{s, t \in V} \frac{\beta_{s, t}}{d(s, t)}
$$

Figure. 6 illustrates the technique of 1-way random walk from $s$ to $t$. $W_{s}$ terminates when it reaches $t . W_{s}$ is indicated by the red walk. The source $s$ and target $t$ is denoted by the red dots. The blue dots indicate the other vertices in the network.

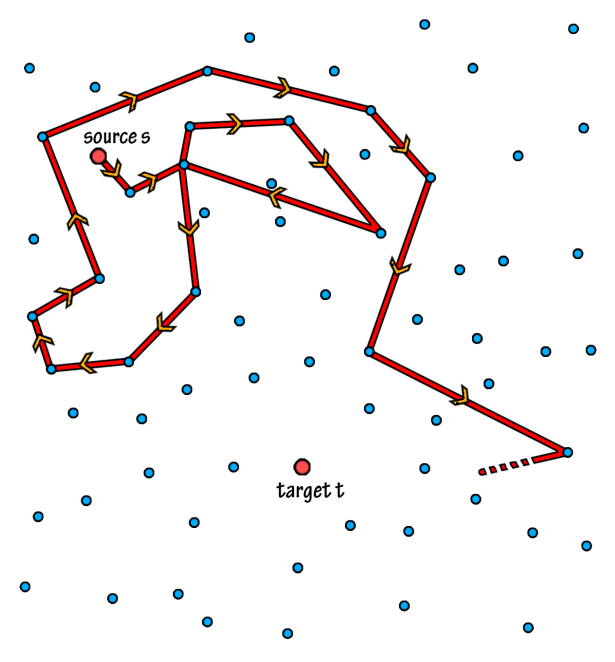

Figure 6: 1-way Random Walk

\subsubsection{2-Way Random Walk}

After taking random walks $W_{s}$ and $W_{t}$ from $s$ and $t$ simultaneously until the two walks intersect, one can find a path from $s$ to $t$.

This idea is similar to the one implemented in the learning phase in Section 4.1. Let $\gamma_{s, t}$ denote the length of the path thus obtained. Let $\gamma$ be the average ratio of length of 2-way Random Walk and the length of the shortest path taken over all the unordered vertex pairs $(s, t)$, such that $s, t \in V$.

$$
\gamma=\frac{1}{\left(\begin{array}{c}
|V| \\
2
\end{array}\right)} \sum_{s, t \in V} \frac{\gamma_{s, t}}{d(s, t)}
$$

Figure 7 illustrates the technique of 2-way random walk between $s$ and $t$. $W_{s}$ and $W_{t}$ are constructed simultaneously until they intersect. The intersection point of the two walks is indicated by $H . W_{s}$ and $W_{t}$ are indicated by the red walk and green walk respectively. Source $s$ and the target $t$ are denoted by the red dots. 


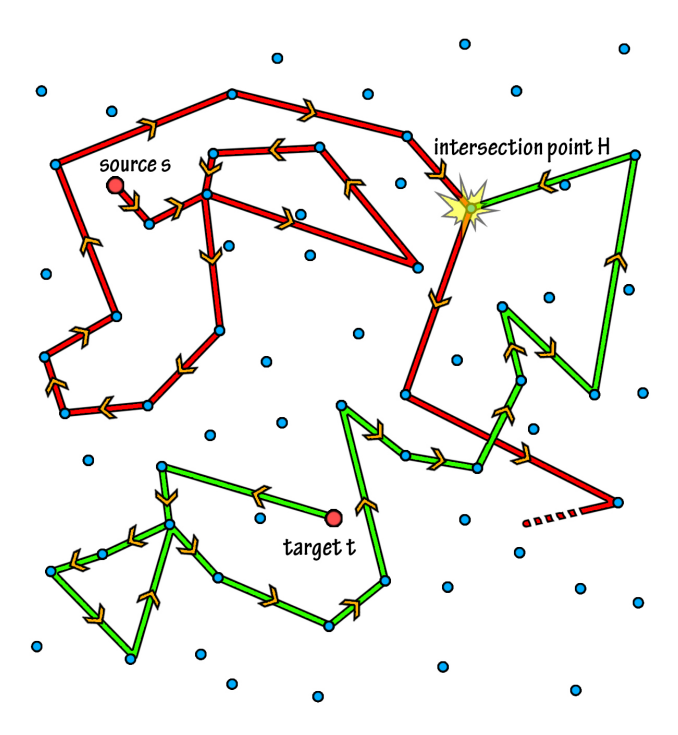

Figure 7: 2-Way Random Walk

\subsection{Comparison between PCA and Degree Based Navigation (Lada A. Adamic)}

Degree based navigation assumes that we have only local knowledge of the network. To pass a message from a source to a target, the source node passes the message to the neighbor with the highest degree. This process continues till the target is found. However, the message may not be passed to a node already visited, if it has other available neighbors. If there are no available neighbors, the message may be passed to a node that is already visited.

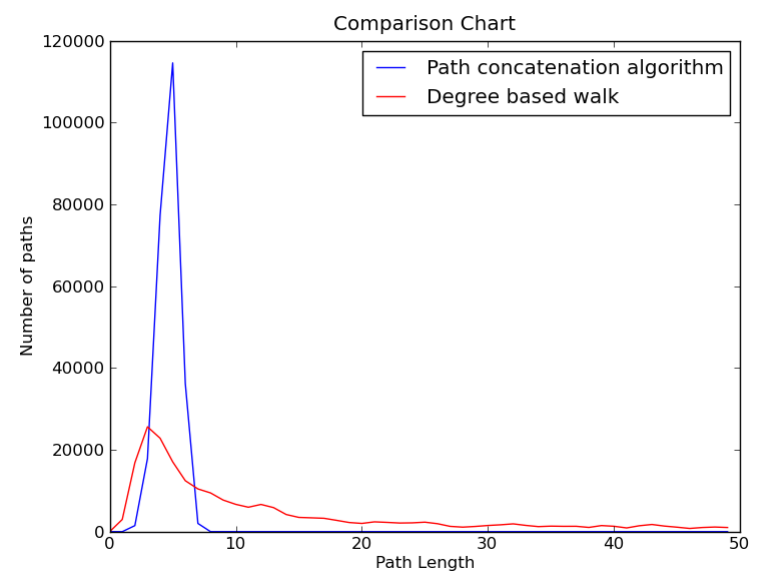

Figure 8: Comparison between PCA and Degree Based Navigation

We compare the path lengths obtained from this type of navigation to the path lengths obtained using PCA for a Scale-Free network of 500 nodes, considering all the vertex pairs. Figure 8 illustrates the empirical results that we have obtained.The average path length for PCA and degree based navigation was found to be 4 and 24 respectively.

As evident from the plot, degree based navigation yields paths of much greater lengths than the PCA paths. This is due to the fact that the degree based approach spends a lot of hops between nodes of high degree after a brief initial period of exploring fresh nodes.

\subsubsection{Path Concatenation Algorithm}

Let $\delta_{s, t}$ denote the length of the path from $s$ to $t$ obtained after executing the path concatenation algorithm. Let $\delta$ be the average ratio of length of the path obtained by the path concatenation algorithm and the length of the shortest path, taken over all the unordered vertex pairs $(s, t)$, such that $s, t \in V$.

$$
\delta=\frac{1}{\left(\begin{array}{c}
|V| \\
2
\end{array}\right)} \sum_{s, t \in V(G)} \frac{\delta_{s, t}}{d(s, t)}
$$

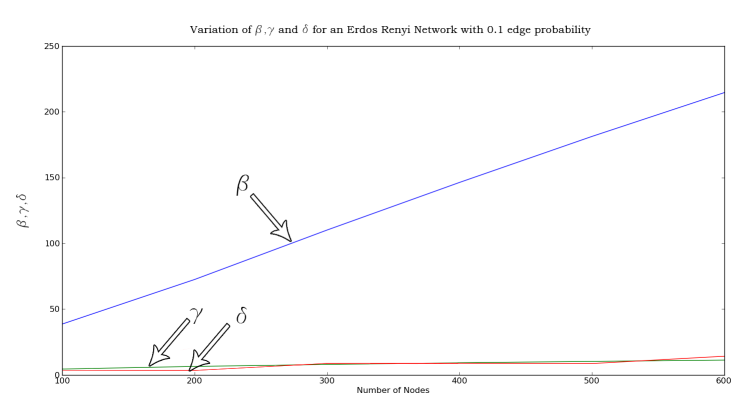

Figure 9: Plot of $\beta, \gamma$ and $\delta$ versus the number of vertices for an Erdos-Renyi network with an edge probability of 0.1

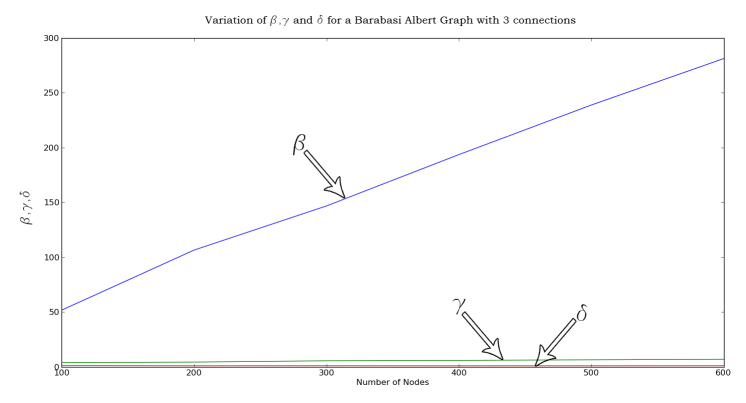

Figure 10: Plot of $\beta, \gamma$ and $\delta$ versus the number of vertices for a Barabasi-Albert Graph with 3 connections

Figure. 9 is a plot of variation of $\beta, \gamma$ and $\delta$ versus the number of vertices for an Erdos-Renyi network with an edge probability of 0.1 . Figure. 10 is a plot of variation of $\beta, \gamma$ and $\delta$ versus the number of vertices for a scale free network with 4 connections. The blue curve indicates $\beta$, green curve indicates $\gamma$ and the red curve indicates $\delta$. From the plots, it is clear that the $\delta$-curve always lies below the $\beta$ curve. This implies that, the greedy navigation performs better than 1way Random Walk.

In case of Erdos-Renyi networks, the $\delta$ curve and the $\gamma$ curve lie very close to each other, and also intersect at certain 


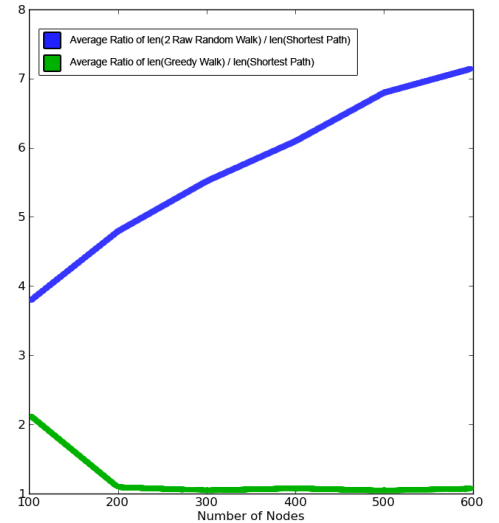

Figure 11: This plot is a zoomed version of previous plot in Figure. 10 of $\gamma$ and $\delta$ versus the number of vertices for a Scale-free Graph with 4 connections

points in the plot. Hence, the performance of the proposed algorithm is as good as a 2 -way random walk technique.

In case of scale-free graphs, the $\delta$ curve always lies below that of the $\gamma$ curve. Hence, the performance of the greedy technique is better than that of the 2 -way random walk technique.

\subsection{Hotspot Distribution for Erdos-Renyi and Scale-free graphs}

The hotspot set we introduced exhibit some characteristic features. We note that the proposed path concatenation algorithm doesn't perform significantly better than the 2way random walk. The reason being that the Erdos-Renyi graphs do not contain vertices of comparatively higher degree than the rest of the vertices in the graph. Whereas in case of a scale-free graph, some vertices have very high degree (hubs) while the rest have comparatively low degree. These $h u b s$ form valid candidates for the hotspot set. They can be reached in a few hops from any vertex in the graph, thus making the PCA an efficient strategy.

Hotspot Distribution for an Erdos Renyi Graph G(1000, 0.3) over several trials
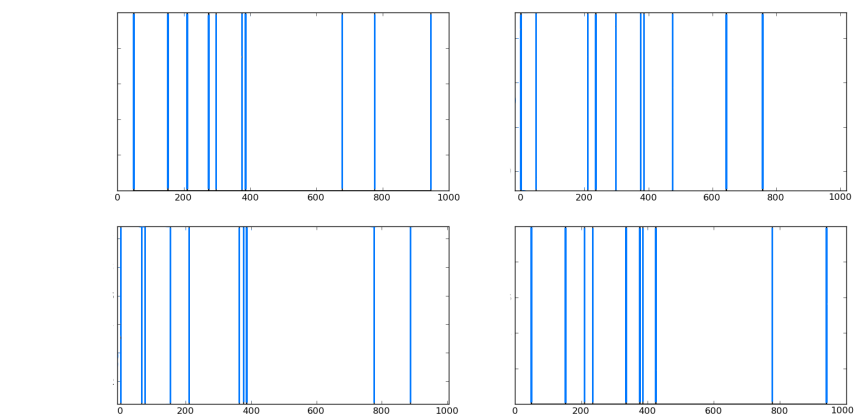

Hotspot Occurance

Figure 12: Hotspot Distribution for an Erdos-Renyi Graph

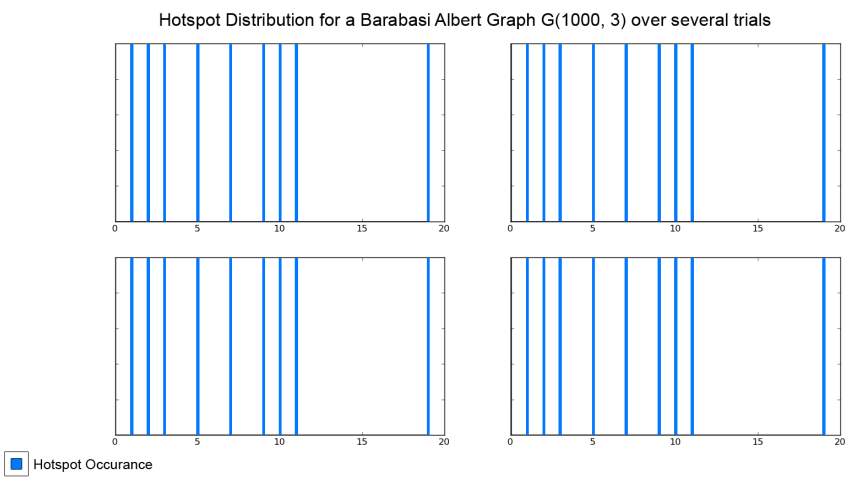

Figure 13: Hotspot Distribution for a Scale-free Graph

Fixing an Erdos-Renyi graph $G$ on 1000 vertices with probability $p=0.3$, as we run the learning phase of the PCA algorithm 4 times on the same graph, we note that the hotspot sets are different each time we run the algorithm. The top hotspot set is shown in Figure. 12, the x-axis denotes the vertex name and the vertical lines represent the hotspot. E.g. if a vertex, say 306, has a vertical line, it means that the vertex is chosen as a hotspot.

Repeating the same procedure on a scale-free graph, we note that the top hotspot set remains more or less the same as shown in Figure. 13

\section{CENTER-STRATEGIC PATHS ON SCALE FREE NETWORKS}

Given a path $\left(v_{1}, v_{2}, v_{3}, \ldots, v_{k}\right)$ with closeness centrality values $\left(c_{C}\left(v_{1}\right), c_{C}\left(v_{2}\right), \ldots, c_{C}\left(v_{k}\right)\right)$ as defined in Section. 3, we compute the ranking of vertices $\left(R\left(v_{1}\right), R\left(v_{2}\right), R\left(v_{3}\right), \ldots, R\left(v_{k}\right)\right)$. By rank-plot of this path, we mean a plot of the ranks $\left(R\left(v_{1}\right), R\left(v_{2}\right), R\left(v_{3}\right), \ldots, R\left(v_{k}\right)\right)$. If the rank-plot of a path has no more than one maxima, we call such a path a centerstrategic path.

The algorithm presented in this article is inspired by the strategy adapted by humans to learn the centers of a network and navigate in a center-strategic way. The presented algorithm resonates with the technique used by humans. We establish this fact by showing that the PCA algorithm yields center-strategic paths on scale free networks.

Consider a scale-free graph $G(V, E)$. Let $k$ denote the number of iterations (or trials) during the navigation phase of the path concatenation algorithm. After $k=100$, for every $k$, we jump to navigation phase, take ${ }^{|V|} C_{2}$ greedy traversals (as explained in Section. 4.2 between all vertex pairs and check for the number of paths that are center-strategic. The ratio of the number of center-strategic paths to the number of greedy traversals ${ }^{|V|} C_{2}$ is denoted by $\psi$.

Figure. 14 shows the variation of $\psi$ as $\mathrm{k}$ increases. We note that the path concatenation algorithm yields centerstrategic paths $80 \%$ of the times on scale free networks. 


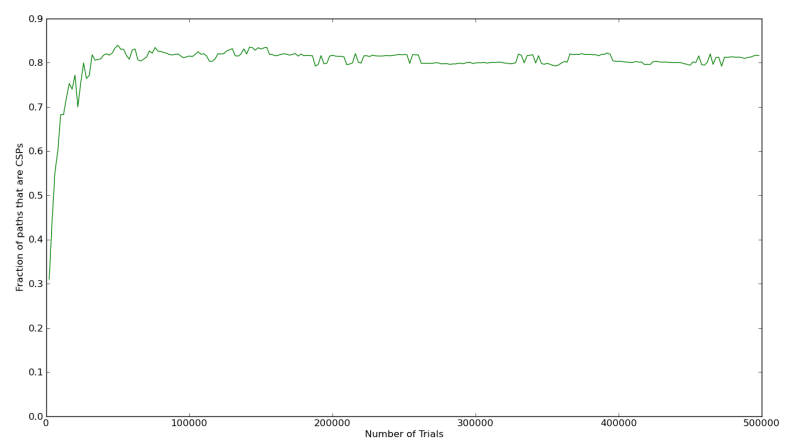

Figure 14: Center Strategic Paths Using Path Concatenation Algorithm

\section{CONCLUSION}

Motivated by the strategy adopted by humans to navigate in an unknown environment, we presented in this paper, an algorithm which simulates human navigation. We showed that the algorithm performs better than the 1-way and 2-way random walk technique. We further showed that the proposed algorithm generates center-strategic paths on scale-free networks.

A possible further work would be to study the convergence of the learning phase of the algorithm. One can start with the basic graph structures like the paths, grids, trees and study the hotspot distribution. It would also be interesting to classify the networks on which the algorithm performs well and those on which it does not. One can study the performance of PCA algorithm on real - world networks as compared to other other navigational techniques.

\section{REFERENCES}

[1] L. A. Adamic and E. Adar. How to search a social network. Social Networks, 27:2005, 2005.

[2] L. A. Adamic, R. M. Lukose, and B. A. Huberman. Local search in unstructured networks. In Handbook of Graphs and Networks, pages 295-317. Wiley-VCH, 2003.

[3] L. A. Adamic, R. M. Lukose, A. R. Puniyani, and B. A. Huberman. Search in power-law networks. Physical Review E, 64(4):046135+, September 2001.

[4] R. Albert and A. L. Barabási. Statistical mechanics of complex networks. Reviews of Modern Physics, 74(1):47-97, January 2002.

[5] D. O. Cajueiro. Optimal navigation in complex networks. Physical Review E, 79(4):046103+, April 2009.

[6] D. O. Cajueiro and R. F. S. Andrade. Learning paths in complex networks. EPL (Europhysics Letters), 87(5):58004, 2009.

[7] A. Crespo and H. Garcia-molina. Routing indices for peer-to-peer systems, 2002.

[8] F. F. Dragan and M. Matamala. Navigating in a graph by aid of its spanning tree metric. 25(1):306-332, 2011.

[9] P. Erdös and A. Renyi. On the evolution of random graphs. Publications of the Mathematical Institute of the Hungarian Academy of Sciences, 5:17-61, 1960.

[10] P. Fraigniaud. Small worlds as navigable augmented networks: model, analysis, and validation. In Proceedings of the 15th annual European conference on Algorithms, ESA'07, pages 2-11, Berlin, Heidelberg, 2007. Springer-Verlag.

[11] C. Gavoille. A survey on interval routing. Theoretical Computer Science, 245:785-796, 1999.

[12] C. Gavoille. Routing in distributed networks: overview and open problems. SIGACT News, 32:36-52, March 2001.

[13] C. Gavoille, D. Peleg, S. Pérennes, and R. Raz. Distance labeling in graphs. Journal of Algorithms, 53(1):85-112, 2004.

[14] S. Giordano, I. Stojmenovic, and L. Blazevic. Position based routing algorithms for ad hoc networks: A taxonomy. In Ad Hoc Wireless Networking, pages 103-136. Kluwer, 2001.

[15] R. P. Grimaldi. Discrete and Combinatorial Mathematics: An Applied Introduction, Fifth Edition. Addison Wesley, 5 edition, July 2003.

[16] Sudarshan Iyengar, Nina Zweig, Abhiram Natarajan, and Veni Madhavan. A network analysis approach to understand human-wayfinding problem. In CogSci 2011 Proceedings, number 33, pages 2836-2841, July 2011.

[17] B. J. Kim, C. N. Yoon, S. K. Han, and H. Jeong. Path finding strategies in scale-free networks. Phys Rev E Stat Nonlin Soft Matter Phys, 65(2 Pt 2), February 2002.

[18] J. Kleinberg. Navigation in a small world. Nature, 406:845, 2000.

[19] J. Kleinberg. Complex Networks and Decentralized Search Algorithms. In In Proceedings of the International Congress of Mathematicians (ICM, 2006.

[20] Jon Kleinberg. The Small-World Phenomenon: An Algorithmic Perspective. In Proceedings of the 32nd ACM Symposium on Theory of Computing, 2000.

[21] D. Liben-Nowell, J. Novak, R. Kumar, P. Raghavan, and A. Tomkins. Geographic routing in social networks. Proceedings of the National Academy of Sciences of the United States of America, 102(33):11623-11628, August 2005.

[22] S. Milgram. The Small World Problem. Psychology Today, 1:61-67, 1967.

[23] D. Peleg. Distributed computing: a locality-sensitive approach. Society for Industrial and Applied Mathematics, Philadelphia, PA, USA, 2000.

[24] G. Sabidussi. The centrality index of a graph. Psychometrika, 31, 1966.

[25] O. Simsek and D. Jensen. Navigating networks by using homophily and degree. Proceedings of the National Academy of Sciences, 105, 2008.

[26] M. Thorup and U. Zwick. Compact routing schemes. In Proceedings of the thirteenth annual ACM symposium on Parallel algorithms and architectures, SPAA '01, pages 1-10, New York, NY, USA, 2001. ACM. 